\title{
Iterative migration of gravity and gravity gradiometry data at Bathurst Mining Camp
}

\author{
Le Wan and Michael S. Zhdanov \\ Department of Geology and Geophysics \\ University of Utah \\ Salt Lake City, Utah, USA
}

\begin{abstract}
Three-dimensional (3D) inversion of full tensor gradiometry (FTG) data continues to be an active area of research and development. We have recently developed a method of potential field migration, which extends to the case of the potential field the general principles of seismic and electromagnetic migration. This new approach provides a rapid method for direct transformation of observed gravity and gravity gradient data into spatial distributions of density. We demonstrate in this paper that migration can be applied iteratively to get more accurate subsurface distributions of the physical properties of rocks. We show that, the iterative migration is practically equivalent to the basic gradient-type inversion algorithms with one very important difference: the gradient directions on each iteration are determined by migration of the corresponding gravity, and gravity gradient data. This is significant because the last transformation is very well developed in the theory of potential field interpretation. In other words, the iterative migration makes it possible to use the powerful and stable technique of upward continuation for the solution of the inverse problem. We present a model study and a case study for the 3D iterative imaging of FTG data from New Brunswick, Canada.
\end{abstract}

Keywords-migration; gravity gradiometry; imaging; inversion

\section{INTRODUCTION}

Density distribution provides important geophysical information that helps in regional geological studies and mineral and energy resource exploration. In oil and gas exploration, density distribution is used to reduce the nonuniqueness of recovering $3 \mathrm{D}$ velocity models from an acoustic impedance. In mineral exploration, density and magnetic susceptibility models are used to locate mineralization zones. At the same time, determining 3D density distribution from gravity and/or gravity gradiometry data is a very challenging problem. Until recently, iterative 3D inversion of gravity and gravity gradiometry data to $3 \mathrm{D}$ density models was the only practical tool for quantitative interpretation. A number of publications have discussed 3D inversion with smooth (e.g., Li ${ }^{11}$ ) or focusing (e.g., Zhdanov et al. ${ }^{[2]}$ ) regularization. However, an interpretation workflow including 3D inversion could be complicated and time consuming and it is very dependent on the a priori model and constraints used.

In this paper, we develop a novel approach, which is based on the ideas of potential field migration as originally introduced by Zhdanov ${ }^{[3]}$. The principles of this approach were presented in Wan and Zhdanov ${ }^{[4]}$, where it was demonstrated that migration could be described by an action of the adjoint operator on the observed data. When applied to potential fields, migration manifests itself as a special form of downward continuation of the potential field and/or its gradients, which is obtained by relocating the sources of the observed field into the upper half-space as mirror images of the true sources. Contrary to conventional downward continuation of the potential field, downward continuation of the migration field is away from the mirror images of the sources. Therefore, migration is a stable transform, similar to upward continuation. By analogy to iterative electromagnetic migration (e.g., Mehanee and Zhdanov ${ }^{[5]}$; Zhdanov [3][6][7]; Zhdanov and Ueda ${ }^{[8]}$ ), the adjoint operators may be applied iteratively, which results in the iterative migration method. We present a model study and a case study for the 3D iterative migration of the FTG marine gravity gradiometry data from New Brunswick, Canada.

\section{GRAVITY GRADIOMETRY DATA AND MIGRATION DENSITY}

The gravity field, g, can be expressed by the gravity potential $\mathrm{U}(\mathrm{r})$ :

$$
\begin{gathered}
\boldsymbol{g}(\mathbf{r})=\nabla U(\mathbf{r}) \\
U(\mathbf{r})=\gamma \iiint_{D} \rho\left(\mathbf{r}^{\prime}\right) \frac{\mathbf{r}^{\prime}-\mathbf{r}}{\left|\mathbf{r}^{\prime}-\mathbf{r}\right|^{2}} d V^{\prime}
\end{gathered}
$$

where $\gamma$ is the universal gravitational constant $\left(\gamma=6.67384 \times 10^{-}{ }^{11} \mathrm{~m}^{3} / \mathrm{kgs}\right)$ and $\rho$ is the anomalous density distribution within a domain $\mathrm{D}$, where integration is conducted over the variable $\mathrm{r}^{\prime}$.

The second spatial derivatives of the gravity potential U(r),

$$
\boldsymbol{g}_{\alpha \beta}=\frac{\partial^{2} U(\mathbf{r})}{\partial \alpha \partial \beta}, \alpha, \beta=x, y, z
$$

form a symmetric gravity tensor:

$$
g=\left|\begin{array}{lll}
g_{x x} & g_{x y} & g_{x z} \\
g_{y x} & g_{y y} & g_{y z} \\
g_{z x} & g_{z y} & g_{z z}
\end{array}\right|
$$


The expressions for the gravity tensor components can be calculated based on equations (1) and (2):

$$
g_{\alpha \beta}=\gamma \iiint_{D} \frac{\rho\left(\boldsymbol{r}^{\prime}\right)}{\left|\boldsymbol{r}^{\prime}-\boldsymbol{r}\right|^{3}} K_{\alpha \beta}\left(\boldsymbol{r}^{\prime}-\boldsymbol{r}\right) d v^{\prime}
$$

where the kernels, $K_{\alpha \beta}$, are equal to

$K_{\alpha \beta}\left(\mathbf{r}^{\prime}-\mathbf{r}\right)=\left\{\begin{array}{l}3 \frac{\left(\alpha-\alpha^{\prime}\right)}{\left|\mathbf{r}^{\prime}-\mathbf{r}\right|^{2}}, \quad \alpha \neq \beta \\ 3 \frac{(\alpha-\omega)^{2}}{\left|\mathbf{r}^{\prime}-\mathbf{r}\right|^{2}}-1, \quad \alpha=\beta\end{array}, \quad \alpha, \beta=\mathrm{x}, \mathrm{y}, \mathrm{z}\right.$

Following Zhdanov ${ }^{[3]}$ and Wan and Zhdanov ${ }^{[4]}$, the migration density $\rho_{\alpha \beta}^{m}$ can be calculated from the migration gravity field, $g_{\alpha \beta}^{m}(\mathbf{r})$, as follows

$$
\begin{aligned}
& \rho_{\alpha \beta}^{m}(\mathbf{r})=k_{\alpha \beta} \omega_{\alpha \beta}^{-2}(z) g_{\alpha \beta}^{m}(\boldsymbol{r}) \\
& =k_{\alpha \beta} \omega_{\alpha \beta}^{-2}(z) \gamma \iint_{s} \frac{g_{\alpha \beta}(\boldsymbol{r})-g_{\alpha \beta}^{o b s}(\boldsymbol{r})}{\left|\boldsymbol{r}^{\prime}-\boldsymbol{r}\right|^{3}} K_{\alpha \beta}\left(\boldsymbol{r}^{\prime}-\boldsymbol{r}\right) d s
\end{aligned}
$$

where unknown coefficient $k_{\alpha \beta}$ can be determined by a linear line search, and the linear weighting operator $\omega_{\alpha \beta}$ is calculated by the square root of the integrated sensitivity of the gravity gradient field (Wan and Zhdanov ${ }^{[4]}$ ).

\section{ITERATIVE MIGRATION}

The equation (6) can produce a migration image of density distribution in the lower half-space. A better quality migration image can be produced by repeating the migration process iteratively. We begin with the migration of the observed gravity and/or gravity tensor field data to get the density distribution. In order to check the accuracy of our migration imaging, we apply the forward modeling and compute a residual between the observed and predicted data for the given density model. If the residual is smaller than the prescribed accuracy level, we use the migration image as a final density model. In a case where the residual is not small enough, we migrate the residual field and produce the density, $\delta \rho^{m}$, to the original density model using the same analysis which we have applied to the original migration:

$$
\rho_{2}^{m}=\rho_{1}^{m}-\delta \rho_{1}^{m}==\rho_{1}-k_{1}\left(W_{m}^{*} W_{m}\right)^{-1} l_{1}
$$

where $\delta \rho_{1}^{m}$ stands for the migration image obtained by the residual field migration, equation (6).
A general scheme for iterative migration can be described by the following formula:

$$
\rho_{n+1}^{m}=\rho_{n}^{m}-\delta \rho_{n}^{m}==\rho_{n}-k_{n}\left(W_{m}^{*} W_{m}\right)^{-1} l_{n}
$$

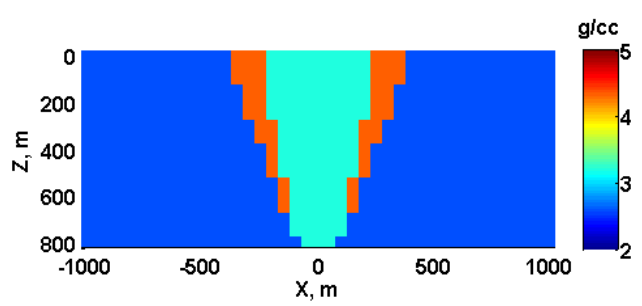

Fig.1 Vertical cross section of the density model.

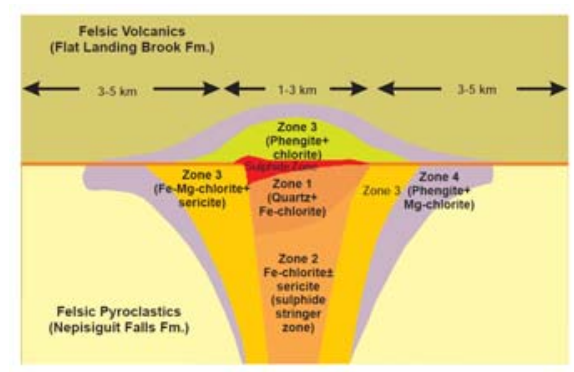

Fig.2 A schematic composite section through a VMS alteration system in the Bathurst camp as an example of a VMS proximal alteration zone metamorphosed to greenschist-grade mineral assemblages. From Goodfellow et al. (2003).
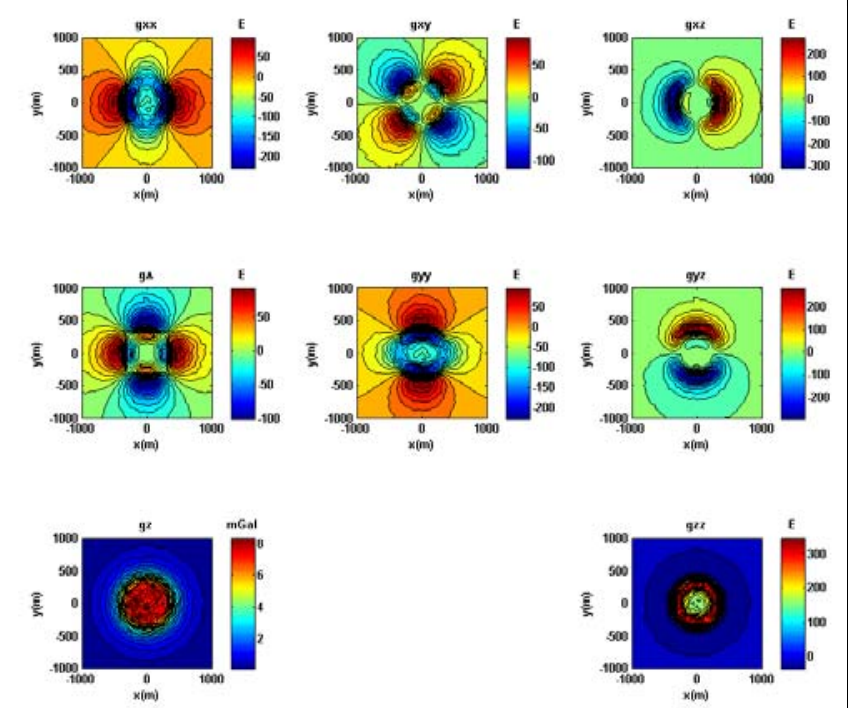
model.

Fig.3 Synthetic gravity and gravity gradient data for VMS deposit

where $l_{n}$ is a gradient direction on the nth iteration, computed using the following integral

$$
l_{n}(\rho)=\gamma \iint_{s} \frac{g_{\alpha \beta}(r)-g_{\alpha \beta}^{o b s}(r)}{\left|r^{\prime}-r\right|^{3}} K_{\alpha \beta}\left(r^{\prime}-r\right) d s
$$


where $g_{\alpha \beta}^{n}(\boldsymbol{r})$ are the predicted gravity gradient fields on the $n$th iteration.

The iterative migration is terminated when the residual field becomes smaller than the required accuracy level of the data fitting.

Similar to iterative inversion, iterative migration can be implemented with regularization (Zhdanov ${ }^{[3]}$ ). This also allows us to apply both the smooth and focusing stabilizers expressions (Wan and Zhdanov ${ }^{[4]}$ ).

\section{MOdEL STUDY}

We have examined the effectiveness of the iterative migration using synthetic gravity and gravity gradiometry data computed for a simple model, shown in Figures 1. The model represents a simplified model of volcanogenic massive sulfide (VMS) deposits (see Fig.2). It has three different density values, from the center to outside: $3.2 \mathrm{~g} / \mathrm{cm}^{3}$ (felsic), $4.3 \mathrm{~g} / \mathrm{cm}^{3}$

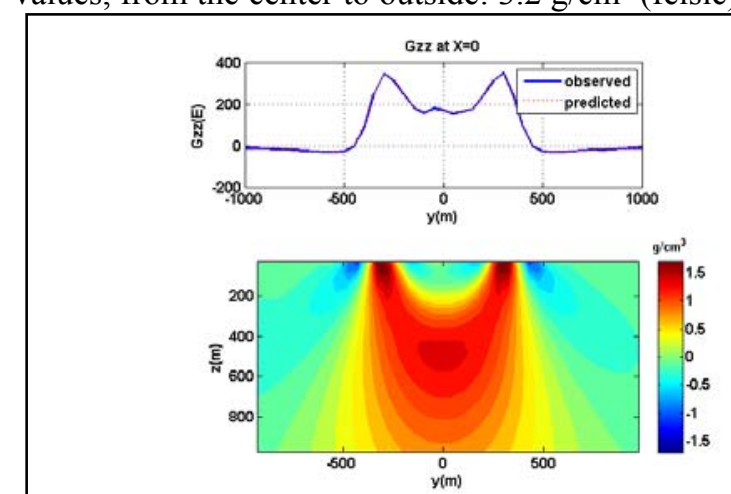

Fig.4 Results of iterative migration with smooth stabilizer for $g_{z z}$ component. The bottom panel shows the migration density distribution in the center cross section, while the top panel presents the profiles of the observed and predicted data.
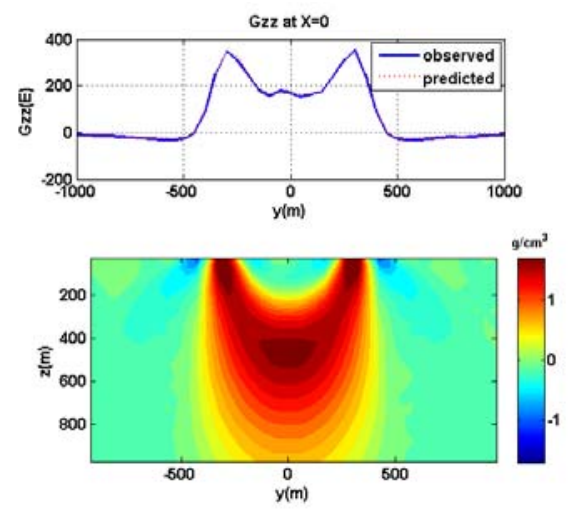

Fig.5 Results of iterative migration with focusing stabilizer for $g_{z z}$ component. The bottom panel shows the migration density distribution in the center cross section, while the top panel presents the profiles of the observed and predicted data.

(mafic), and $2.6 \mathrm{~g} / \mathrm{cm}^{3}$ (host rock), respectively. Figures 1 show the center cross section of the model. The observation surface is located $80 \mathrm{~m}$ above the ground. The survey area extends from $-1000 \mathrm{~m}$ to $1000 \mathrm{~m}$ along the $\mathrm{x}$ direction and from $-1000 \mathrm{~m}$ to $1000 \mathrm{~m}$ along the $\mathrm{y}$ direction. There are $41 \mathrm{x}$ $41=1681$ data points for each component. For testing the algorithm, the synthetic observed data generated for this model were contaminated by $5 \%$ random noise.

Fig.3 shows the synthetic observed data computer simulated for this model. One can see that the anomaly is affected by the noise and becomes asymmetric.

The migration domain was selected from $-1000 \mathrm{~m}$ to 1000 $\mathrm{m}$ in the $\mathrm{x}$ direction, from $-1000 \mathrm{~m}$ to $1000 \mathrm{~m}$ in the $\mathrm{y}$ direction, and from 0 mto $1000 \mathrm{~m}$ in the $\mathrm{z}$ direction.

We have applied the iterative migration with the smooth stabilizer to all of components. The bottom panel in Fig.4 shows the migration density distribution in the center cross section for gzz component, while the top panel presents the profiles of the observed and predicted data. One can see that the migration result locates the target and the data fitting is very good.

We have also applied iterative migration with focusing stabilizer. Fig.5 shows the migration density distribution in the center cross section, obtained by iterative migration with focusing stabilizer for gzz component. One can see that the iterative migration with focusing stabilizer recovers the true density value of the target.

Case study: FTG survey from New Brunswick, Canada

We illustrate our method with an example from the Bathurst Mining Camp in New Brunswick, Canada, where at least $35 \mathrm{~Pb}-\mathrm{Zn}-\mathrm{Cu}-\mathrm{Ag}$ type VMS deposits and over 100 known mineral occurrences have been discovered since the early 1950 s.

The structural geology of the camp is complex, with five groups of folds identified. It has been suggested that most of the Tetagouche volcanic rocks are of basin-margin origin, deposited on a rifting continental crust. It follows that many of the VMS deposits are associated with tuffite and silicic volcanic rocks of the Nepisiguit Falls and Flat Landing Brook formations of the Tetagouche Group (Van Staal ${ }^{[5]}$; Lentz ${ }^{[6]}$ ). Typically, the VMS deposits have a density of about $4 \mathrm{~g} / \mathrm{cc}$, whereas the host rocks (sediments, felsic tuffs, or their metamorphic equivalents) have densities between 2.7 and 2.8 $\mathrm{g} / \mathrm{cc}$ (Dransfield et $\mathrm{al}^{[7]}$ ).

On behalf of Noranda (now Glencore), SLAM Exploration, and the Government of New Brunswick, Bell Geospace acquired 15,500 line $\mathrm{km}$ of Air-FTG full tensor gravity gradiometry data that covered more than 2,755 square $\mathrm{km}$ of the camp during 2004. The survey was flown at $200 \mathrm{~m}$ line spacing with $2000 \mathrm{~m}$ tie lines and $80 \mathrm{~m}$ topographic drape. Bell Geospace subsequently re-processed the Air-FTG data during 2010 using improved terrain correction, leveling, automatic tilt and full tensor noise reduction (FTNR). Figure 19 shows the Air-FTG full tensor gradient data collected in 2004 with the terrain corrections applied. 


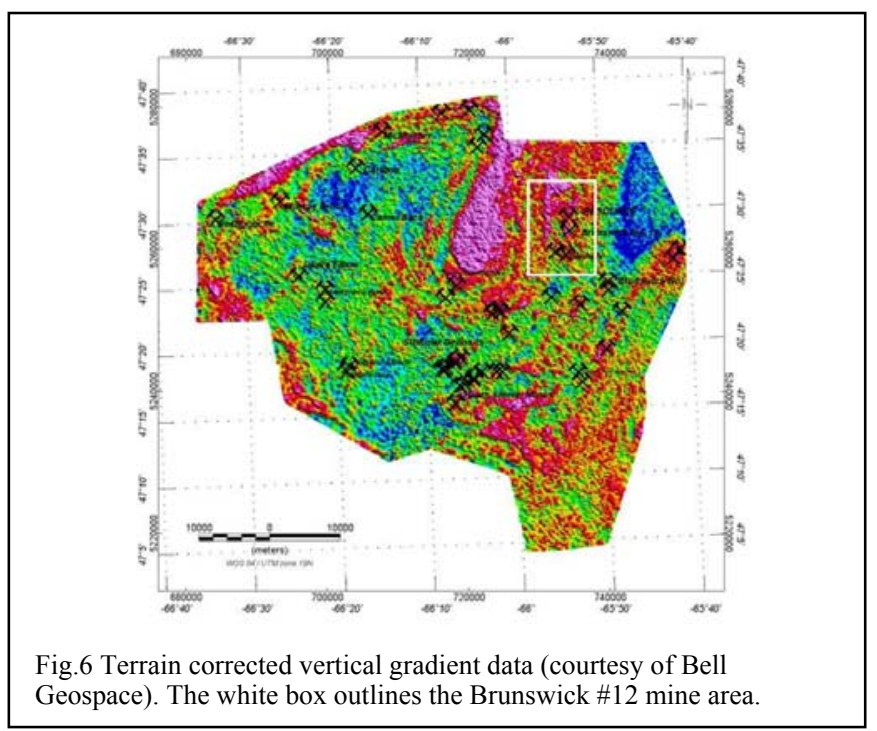

We selected the part of the area around Brunswick \#12 mine, about $10 \mathrm{~km}$ (Eastern) by $13 \mathrm{~km}$ (Northern) in size, shown in Fig.6.

The Brunswick \#12 mine is an underground lead-zinccopper mine in the Bathurst Mining Camp of northern New Brunswick, Canada. It was discovered in January, 1953 and entered production in April, 1964. The Brunswick \#12 ore body is the largest deposit in the Bathurst area and was one of the largest underground zinc mines in the world well into the late 1990s. The supergiant Brunswick \#12 deposit is a volcanogenic massive sulfide (VMS) deposit rich in lead, zinc, and copper. Currently the copper-rich portion of the deposit has not been mined.

The migration domain was $10 \mathrm{~km}$ long in the $\mathrm{x}$ direction (East) and $13 \mathrm{~km}$ long in the $\mathrm{y}$ direction (North), and it extended down to $5 \mathrm{~km}$ in the $\mathrm{z}$ direction. We applied Iterative migration with both smooth and focusing stabilizers.

We have overlapped the density distribution at $500 \mathrm{~m}$ depth produced by iterative migration with a geological map (Fig.7). We can see a reasonable correlation between the migration density model and the known geological features (e.g., inferred thrusts) in the survey area.

\section{CONCLUSION}

Potential field migration is a direct integral transformation of a gravity field and/or gradients into 3D density distributions. We have demonstrated that migration can be applied iteratively to get more accurate subsurface distributions of the physical properties of rocks. We have shown also that, the iterative migration is practically equivalent to the basic gradient-type inversion algorithms with one very important difference: the gradient directions on each iteration are determined by migration of the corresponding gravity, and gravity gradient data, which is equivalent to upward analytical continuation. This is significant because the last transformation is very well developed in the theory of the potential field interpretation. In other words, the iterative migration makes it possible to use the powerful and stable technique of upward continuation for the solution of the inverse problem.

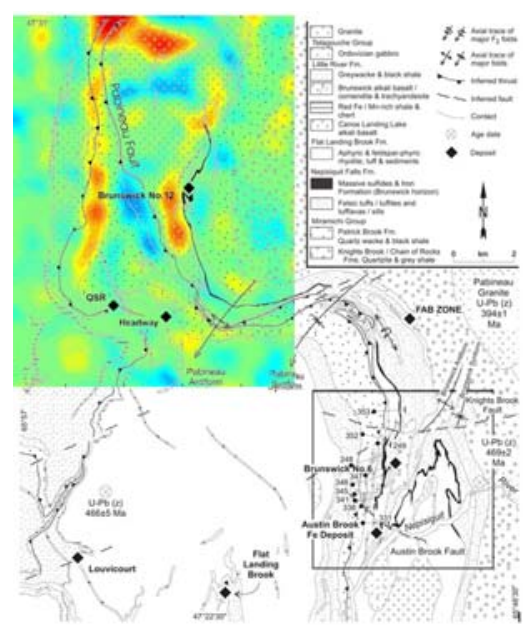

Fig.7 Horizontal cross section of density contrasts (relative to 2.70 $\mathrm{g} / \mathrm{cc}$ ) at $500 \mathrm{~m}$ depth as extracted from the 3D model obtained from migration of the vertical gradient component of Air-FTG data collected over the Brunswick \#12 mine area, superimposed on the surface geology (after Lentz, 1999).

As demonstrated with our model study and case studies of FTG data in New Brunswick, Canada, the results of 3D potential field iterative migration are similar to those obtained from $3 \mathrm{D}$ regularized inversion. The iterative migration can be applied with smooth and focusing stabilizers to produce images with relatively sharp boundaries. Iterative migration also can be applied jointly using different components.

\section{ACKNOWLEDGMENTS}

The authors acknowledge the support of the University of Utah Consortium for Electromagnetic Modeling and Inversion (CEMI), and TechnoImaging for permission to publish. We also acknowledge Bell Geospace for assistance with the Bathurst Mining Camp Air-FTG data released by the Department of Natural Resources of the Government of New Brunswick.

\section{REFERENCE}

[1] Y. Li, "3D inversion of gravity gradiometer data", 71st SEG Annual International Meeting, 2001, San Antonio, Texas, USA, Expanded Abstracts. doi: 10.1190/ 1.1816383.

[2] M. S. Zhdanov, R. G. Ellis, and S. Mukherjee, "Regularized focusing inversion of 3D gravity tensor data", Geophysics, 2004, 69, 925--937. doi:10.1190/1.1778236.

[3] M. S. Zhdanov, Geophysical inverse theory and regularization problems: Elsevier, 2002.

[4] L. Wan and M. S. Zhdanov, "Iterative migration of gravity and gravity gradiometry data", SEG Technical Program Expanded Abstracts 2013: 1211-1215.

[5] C. R. Van Staal, "The Ordovician Tetagouche Group, Bathurst camp, northern New Brunswick, Canada: History, tectonic setting, and distribution of massive sulfide deposits", Exploration and Mining Geology, 1992, 1, 93-103.

[6] D. R. Lentz, "Petrology, geochemistry, and oxygen isotope interpretation of felsic volcanic and related rocks hosting the Brunswick 6 and 12 massive sulfide deposits (Brunswick Belt), Bathurst Mining Camp, New Brunswick, Canada", Economic Geology, 1999, 94, 57-86.

[7] M. Dransfield, "Conforming FALCON gravity and the global gravity anomaly", Geophysical Prospecting, 2010, 58, 469-483 